\title{
DE LA TOXICOMANIE AUX VÉCUS TOXICOMANIAQUES : RÉCITS DE VIE DE DEUX CONSOMMATEURS D'HÉROÏNE
}

\author{
Nelson das Neves Ribeiro, Laurent Chabert
}

Dalloz | «Revue de science criminelle et de droit pénal comparé »

2020/2 $\mathrm{N}^{\circ} 2$ | pages 467 à 486

ISSN 0035-1733

ISBN 9782995520022

DOI 10.3917/rsc.2002.0467

\section{Article disponible en ligne à l'adresse :}

https://www.cairn.info/revue-de-science-criminelle-et-de-droit-penalcompare-2020-2-page-467.htm

Distribution électronique Cairn.info pour Dalloz.

(C) Dalloz. Tous droits réservés pour tous pays.

La reproduction ou représentation de cet article, notamment par photocopie, n'est autorisée que dans les limites des conditions générales d'utilisation du site ou, le cas échéant, des conditions générales de la licence souscrite par votre établissement. Toute autre reproduction ou représentation, en tout ou partie, sous quelque forme et de quelque manière que ce soit, est interdite sauf accord préalable et écrit de l'éditeur, en dehors des cas prévus par la législation en vigueur en France. Il est précisé que son stockage dans une base de données est également interdit. 


\title{
CHRONIQUES
}

\section{Chronique de criminologie}

\author{
Nelson Das Neves Ribeiro
}

Centre de recherches Pénalité, sécurité \& déviances,

Université libre de Bruxelles

\section{Laurent Chabert}

Aspirant F.R.S.-FNRS

Centre de recherches Pénalité, sécurité \& déviances,

Université libre de Bruxelles

\section{De la toxicomanie aux vécus toxicomaniaques :} récits de vie de deux consommateurs d'héroïne

\section{Introduction}

L'Homme est un être incarné, toujours situé dans un rapport au monde, à autrui et à soi. Ce rapport est singulier et dynamique, inscrit et façonné par la biographie de son sujet en fonction du sens qu'il lui donne. Aussi, les substances psychoactives ont universellement conquis ce rapport; en tous temps et en tous lieux, l'Homme a nourri ses interactions extérieures et intersubjectives dans une quête de plaisir ou un besoin d'atténuer ses souffrances. La « drogue » n'est donc «pas seulement un produit lque l'on fume, boit, inhale, s'injecte ou mange), c'est aussi une relation à soi et à autrui, c'est un mode d'être ou une série de modes d'êtres » (Ehrenberg et Schiray, 1992, 11).

À partir du XIXe siècle, ce rapport subjectif va être appréhendé médicalement, d'abord, à travers une lecture «pharmacocentriste» faisant reposer la relation usager-drogue sur les seules propriétés pharmacologiques du produit consommé (Decorte, 2010). Le paradigme pénal associera, ensuite, les drogues illicites à la marginalité et à la délinquance (Brochu, 1997), les propriétés de la substance consommée étant censées mener l'usager à vivre hors-société. Produit d'une construction politique, légale et médiatique reposant sur son association à la marginalité sociale (Faugeron et Kokoreff, 2002), au crime et à l'aliénation (Toro, 1998), le « toxico-délinquant » est alors inexorablement porteur d'une pathologie individuelle et sociale. Si la toxicomanie est une construction (Kaminski, 1990), elle produit donc des effets et contamine les personnes qui en sont étiquetées : elles se voient ainsi réduites à leurs traits toxicomaniaques et affublées d'un stigmate qui les discrédite en tant qu'acteur social. Figure de l'exclusion sociale et 
morale, le toxicomane est alors mis au rang de l'anormalité sous le poids des paradigmes dominants.

Mais peut-on réduire la toxicomanie au seul champ du pathologique ? Pour Canguilhem (1975), définir un comportement comme étant normal ou, à l'inverse, pathologique, relève d'une activité normative, un jugement de valeur en rapport à un idéal que l'on cherche à atteindre. En ce sens, l'auteur distingue anomalie et anormal, ce dernier terme étant entendu dans le même sens que pathologique. L'anomalie se définit comme « toute déviation du type spécifique ou en d'autres termes toute particularité organique que présente un individu comparé à la grande majorité des individus de son espèce, de son âge, de son sexe » (Geoffroy Saint-Hilaire, cité par Canguilhem, 1975,82 ). L'anomal désigne dès lors un fait, une réalité descriptive et un écart statistique sans référence à une quelconque valeur : l'anomalie c'est l'insolite, l'inaccoutumé. À l'inverse, l'anormal, ou le pathologique, renvoie à une anomalie jugée négativement, c'est-à-dire comme étant - « relativement à l'activité de l'individu, et donc à la représentation qu'il se fait de sa valeur et de sa destinée » (Canguilhem, 1975, 87) - une infirmité, un obstacle à l'exercice des fonctions vécu comme une déchéance irrémédiable. Deux conséquences en découlent. Premièrement, le pathologique est toujours un construit social, c'est-à-dire un rapport aux valeurs que la collectivité se donne comme idéal à atteindre. Deuxièmement, le pathologique est une activité éprouvée, qui ne peut se mesurer à l'aune de critères descriptifs et quantitatifs mais bien d'une qualité, à savoir un « sentiment direct et concret de souffrance et d'impuissance, sentiment de vie contrariée » (Canguilhem, 1975, 85). En définitive, « on est donc malade non seulement par référence aux autres, mais par rapport à soi » (Canguilhem, 1975, 87).
Le problème survient lorsqu'une certaine médecine psychiatrique positiviste, aujourd'hui hégémonique et calquant son modèle sur la médecine expérimentale, a prétendu pouvoir se dégager du champ axiomatique pour élaborer un savoir dit « objectif » sur la maladie. S'en suit une confusion sémantique entre l'anomalie et le pathologique : tout écart à la norme statistique - les caractéristiques que l'on retrouve en grande majorité dans une population - est ainsi susceptible de constituer autant de signes morbides, et ce en fonction de valeurs sociétales qui sont explicitement déniées. La conséquence directe d'une telle approche est d'essentialiser la maladie, c'est-à-dire considérer qu'elle existe « en soi », objet parfaitement mesurable, dénombrable et discernable, sans prise en compte de l'environnement dans lequel elle s'insère ou du vécu de celui qui l'éprouve. Sans prétendre ranimer une critique déjà foisonnante concernant la « bible » du diagnostic (Adam, 2012 ; Frances, 2013 ; Kirk et Kutchins, 1998) qu'incarne le Manuel Statistique et Diagnostic des Troubles Mentaux (DSM), aujourd'hui à la cinquième version, nous ne pouvions faire l'impasse sur un bref aperçu des critères retenus pour diagnostiquer le Substance-Related Disorders (American Psychiatric Association, 2013, 483-589). D'une part, une grande majorité de ces critères sont quantitatifs, descriptifs et extrinsèques, c'est-à-dire des caractéristiques formelles de la dépendance: quantité plus importante ; temps passé pour obtenir le produit ; utilisation répétée; tolérance au produit plus forte. L'addition des critères définit le niveau d'addiction sans que l'on ne comprenne la manière dont ces critères peuvent interagir. D'autre part, l'essentialisation de l'addiction due au produit implique que toute référence à la souffrance est toujours envisagée comme étant causée par le produit, selon des schèmes mécanicistes et déterministes qui ne laissent plus de place au sujet, sa capacité à se 
distancer de l'addiction et la manière dont le produit peut aussi répondre à une souffrance déjà présente. Enfin, les critères sont décontextualisés, et lorsqu'il est fait référence à l'environnement c'est toujours dans l'optique de mesurer l'impact de l'utilisation du produit sur celui-ci mais jamais d'interroger en quoi cet environnement peut altérer la consommation du produit.

À l'inverse d'une approche nosologique aussi réductrice et catégorisante, sorte de mise en système de l'existence qui, en voulant ramener l'humain à des relations mécaniques et intelligibles délaisse la grande variabilité des vécus des usagers de produits psychoactifs, nous souhaitons dans cet article privilégier une approche qualitative et non stigmatisante du vécu toxicomaniaque. Pour ce faire, notre ancrage se veut phénoménologique, c'est-à-dire qu'il laisse une large part au vécu de deux usagers d'héroïne rencontrés dans le cadre d'entretiens de type récit de vie ${ }^{1}$ en tant que «narration subjective à d'autres personnes de sa propre vie, ou fragments de sa vie » (Rhéaume, 2008, 63). Outil fondamentalement subjectif (Burrick, 2010, 8), le récit de vie nous permet ainsi d'interroger, dans le cas des deux usagers rencontrés, le sens que revêt leur consommation à l'aune de leur rapport à eux-mêmes, à l'autre et au monde. Le choix de mettre en dialectique seulement deux usagers s'est imposé dans la mesure où ils nous semblaient incarner des trajectoires toxicomaniaques diamétralement opposées et ainsi même paradigmatiques: non pas en termes de quantité puisqu'ils témoignent tous deux d'une consommation élevée d'héroïne, mais en termes de qualité, d'une souffrance ressentie par l'une des interrogés qui l'inscrit dans un vécu pathologique, ce qui n'est pas le cas pour l'autre consommateur. Sans prétendre épuiser ainsi la variabilité $d u$ vécu des usagers de drogues, nous faisons le pari que la mise en tension dialectique de ces deux témoignages, où les paroles de l'un éclairent les problématiques de l'autre, puisse nourrir un savoir plus intense, moins "extérieur », sur la signification de la consommation de drogues dites « dures » dans des trajectoires individuelles pourtant si singulières. De tels témoignages mettent dès lors en lumière l'idée que ce n'est pas le produit qui est créateur de souffrance, inscrivant d'emblée son utilisateur dans le champ du pathologique, mais que le recours au produit peut en revanche s'inscrire comme une forme d'adaptation à un environnement qui est, lui, pathogène, afin d'introduire un nouveau rapport au monde.

\section{I - Trajectoires toxicomaniaques paradigmatiques}

Afin que le lecteur puisse situer notre analyse dans la trajectoire de vie et de consommation de ces deux usagers, il convient d'en faire une brève présentation. Julie est une femme d'une quarantaine d'années. Elle est la plus jeune de deux sœurs. Ses premières expériences avec des psychotropes ont lieu lorsqu'elle a 10 ans: "Je piquais les Valium à ma mère, je les écrasais, je les mettais en trait, je les sniffais ». Par la suite, Julie commence à consommer de l'héroïne en shoot à 15 ans avec son meilleur ami de l'époque. Elle abandonne sa scolarité en cinquième professionnelle: «Soit je m'endormais parce que j'avais pris trop d'héro la veille avec François, soit il me disait "tiens, rendez-vous après le diner devant, dans le parking et on se casse, on va chez moi". Donc, j'ai raté la plupart de l'école, et en cinquième professionnelle, j'ai arrêté ». Lorsqu'elle a 17 ans, son meilleur ami meurt d'une overdose à ses côtés. À

(1) Les deux usagers ont été rencontrés dans le cadre de la recherche doctorale en cours de Nelson das Neves Ribeiro, "Impacts et articulations des contrôles sociaux dans les trajectoires de consommation des usagers de drogue ". 
ses 18 ans, elle se marie, tout en continuant à consommer de l'héroïne. Elle a un enfant de cette première union et fait une dépression post-partum : «J'avais tout, tout, tout! Puis je suis tombée enceinte... II n'aurait pas fallu ». Lorsque son fils a 11 mois, elle demande le divorce, avoue sa toxicomanie à son mari et à ses parents et perd la garde de son enfant. Suite à son divorce, elle rentre de plus en plus dans le monde de la drogue tandis qu'elle se coupe du monde conventionnel. Son entourage est constitué de dealers et de consommateurs, et elle prend de fortes doses de cocaïne et d'héroïne qu'elle consomme en sniff, en inhalation ${ }^{2}$ et en injection. Elle fait ainsi sa première overdose vers 25 ans. Elle en fera cinq tout au long de son parcours de consommation dont la dernière trois mois avant notre entretien. Suite à sa première overdose, elle commence un traitement de substitution qu'elle prend toujours. Elle a un deuxième enfant avec un compagnon consommateur de qui elle se sépare avant la naissance. Son deuxième enfant est placé en famille d'accueil à 3 ans à la suite d'un accident : «On était tellement fusionnels que quand il est rentré à l'école, j'ai fait comme une petite dépression et j'ai repris un peu d'héro. Et je l'ai laissé un peu derrière moi jouer, et il est tombé ». Julie vit de petits remplacements comme serveuse dans des cafés et, surtout, de la prostitution qui lui permet de payer sa consommation. La vie de Julie est marquée par la violence dont elle garde les traces sur son corps. Elle subit notamment quatre viols. Julie oscille à présent entre consommation et cures de désintoxication. Au moment de l'entretien, elle n'a plus de contacts ni avec ses enfants, ni avec sa sœur, ni avec son père, et sa mère est décédée. Julie est hébergée dans une maison d'accueil pour femmes en difficulté à Bruxelles et attend son entrée en cure de désintoxication.

David est un homme d'une cinquantaine d'années issu d'une famille d'émigrés espagnols. Il fait partie d'une fratrie de deux garcons et une fille. Il suit une scolarité classique avec un seul redoublement en cinquième secondaire et commence à consommer du cannabis ponctuellement aux alentours de 15 ans. Son usage devient régulier aux alentours de 18 ans, lorsqu'il entame ses études supérieures. À l'université, il connaît deux échecs en candidature et décide de partir en France : " Je prends la route, je vais faire un peu un tour de France à la va-comme-je-te-pousse, à peu près un an de sans-abrisme organisé, j'ai fait la manche. C'était très, très gai. À un moment j'ai vu la limite de tout ça, je suis revenu en Belgique ». À son retour en Belgique, il rencontre un ami qui lui fait goûter l'héroïne, qu'il consomme pendant vingt-cing ans en inhalation. Il réussit pour autant des études supérieures et entame une vie professionnelle stable en parallèle de sa consommation d'héroïne et de cannabis. Il effectue plusieurs voyages en Asie du sud-est et en Afrique où il rencontre sa femme avec qui il a deux enfants. En 2016, il fait un infarctus et décide de diminuer sa consommation d'héroïne qui devient dès lors ponctuelle. Au moment de l'entretien, David n'a plus fumé d'héroïne depuis plusieurs mois et consomme toujours du cannabis quotidiennement. Il habite à Bruxelles, est toujours marié, entretient des contacts réguliers avec ses parents et sa sœur, et son frère est décédé récemment.

Afin de laisser le plus largement possible la parole aux interrogés, à leur vécu et au sens donné à leur consommation, sans pour autant nous conten-

(2) "Ce mode de consommation que l'on nomme aussi "chasser le dragon" consiste à faire chauffer un caillou d'héroïne sur un morceau d'aluminium. La chaleur transforme le produit en goutte de laquelle s'échappera de la fumée qui sera alors inhalée par l'usager. L'inhalation permet de doser plus facilement les quantités qui seront consommées et de consommer par "paliers", ce qui est difficilement possible lors d'une consommation en injection. Les effets étant ressentis très rapidement, selon son état, les effets recherchés et le contexte, I'usager peut décider de poursuivre sa consommation ou non » (Doyen et das Neves Ribeiro, 2019, 143). 
ter d'un article purement descriptif, nous présentons notre propos selon une optique structurale dialectique. Autrement dit, nous allons ainsi voir que plusieurs polarités structurent l'univers des deux usagers interrogés et que la compréhension du sens que revêt pour eux leur toxicomanie implique de saisir les tensions dialectiques entre ces polarités ainsi que la manière dont le sujet parvient ou non à les résoudre. Celles-ci trouvent à s'incarner dans trois dimensions de la vie des usagers rencontrés: leur rapport à soi, à l'autre et au monde.

\section{II - Le rapport à soi : idem, ipse et identité narrative}

Au centre de la problématique du rapport à soi se joue la question de l'identité : qui suis-je? Question ô combien complexe tant la « transparence de soi à soi est impossible » (Ryckel et Delvigne, 2010,230 ) et que le «je » du «qui » nous apparaît comme fragmenté en de multiples instances, faisant de l'Homme cet être «pluriel » (Lahire, 1998) à la fois sujet social, sujet inconscient, sujet existentiel, sujet réflexif et sujet acteur (Gaulejac, 2009). Aussi, face à cette altérité fondamentale du «je», comment penser la "sensation de continuité, de cohérence et d'unité de nous-même » (Ryckel et Delvigne, 2010, 231) ? C'est pour ouvrir la réflexion face à cette épineuse question de la permanence de soi que Paul Ricœur, dans le tome III de Temps et Récit (1985), ainsi que dans Soi-même comme un autre (1990), considère que l'identité se féconde dans la tension dialectique entre deux pôles: l'idem, ou "mêmeté », et l'ipse ou « soi-même ». L'identité-idem renvoie à un principe de permanence de l'être qui ignore le changement dans le temps. Elle trouve dès lors à s'incarner dans le caractère, c'est-à-dire « l'ensemble des marques distinctives qui permettent de réidentifier un individu humain comme étant le même » (Ricœur, 1990, 144). Il s'agit là d'une identité substantielle, structurale et immuable. À l'opposé, l'identité-ipse désigne le pôle dynamique qui inclut «le changement, la mutabilité, dans la cohésion d'une vie » (Ricœur, 1985, 443). C'est dans l'identité-ipse que se forge le sentiment de subjectivité, autrement dit «la reconnaissance qu'a le sujet d'être à l'initiative de ses actes, de son éthique et de ses paroles » (Ryckel et Delvigne, 2010, 232), et dont Ricœur voit le modèle dans la promesse et la fidélité à la parole donnée. Comment, dès lors, articuler le pôle de l'idem et le pôle de l'ipse, permanence dans le temps du maintien du caractère et maintien de soi ? Pour le philosophe, cette articulation s'effectue par la médiation de l'identité narrative, c'est-à-dire une mise en intrigue de sa vie par le récit « qui permet d'intégrer à la permanence dans le temps ce qui paraît en être le contraire sous le régime de l'identité-mêmeté, à savoir la diversité, la variabilité, la discontinuité, l'instabilité » (Ricœur, 1990, 167-168). Autrement dit, l'identité narrative incarne le moment où l'individu, en racontant son histoire, réintègre dans la discordance des événements une unité concordante, un sens, et construit par là son identité. En reliant le temps à la subjectivité par une saisie réflexive, elle est dès lors constitutive de l'ipséité et fait du sujet le lecteur et le scripteur de sa propre vie.

À présent que ces jalons de compréhension de l'identité, et donc du rapport à soi, ont été posés, il est temps de laisser place au récit de vie de Julie et David en vue de saisir de manière plus approfondie, dans la «mise en intrigue » de leur existence, cette articulation entre l'idem et l'ipse. Notons que cette différence trouve aussi à se marquer, dans la pensée existentialiste, par la différence entre l'en-soi et le pour-soi. De la confrontation de leur récit surgit dès lors deux identités narratives diamétra- 
lement opposées. Elles s'échelonnent du pôle de l'existence perdue au pôle de l'existence conquise et mettent en évidence deux modalités de ce rapport à soi, l'une à la voix passive et l'autre à la voix active :

«J'ai une haine... Mais j'ai plein de haine en moi! T'as déjà remarqué ? Pour plein de choses ... (sanglots). Ma vie, ce qu'on m'a fait, ce que j'ai subi. C'est pour ça que je répète à beaucoup des éducatrices: "Qu'est-ce que je fais là ? Qu'est-ce que je fais là ? Pour qui je suis là ?" » (Julie).

" Je suis toute cette merde (sanglots)! Mais si je suis toute cette merde, c'est parce qu'on me l'a infligée. On m'a infligé certaines choses qui m'ont fait devenir ce que je suis maintenant» (Julie).

Julie témoigne ainsi d'un vécu de souffrance et d'une trajectoire de vie jugée à l'aune d'une déchéance irrémédiable. Elle se vit comme étant condamnée, engluée dans un parcours toxicomaniaque dont elle ne peut plus, ou ne veut plus sortir. Aussi ne parvient-elle pas, par le « je » de l'identité narrative, à élaborer un sens à une existence marquée par la perte de contrôle et envisagée comme étant subie plutôt que choisie :

"Quelqu'un qui a touché aux drogues dures ne s'arrête jamais de sa vie! Soit il meurt d'une overdose, soit il meurt d'une pancréatite ou d'un truc lié à son foie ou une hépatite, soit le sida, soit crise cardiaque. Moi je ne crois pas en l'abstinence totale et à vie. Un jour ou l'autre tu auras envie de te redéchirer la tête. S'arrêter totalement et à vie, en tout cas dans mon cas, je n'y crois pas. Je ne suis pas assez forte » (Julie).

On reconnaît là le «mode d'être » (Ricœur, 1990, 358) constitutif de l'idem. Julie s'éprouve dans la permanence de son identité, celle d'une toxicomane que les gens évitent «parce que je suis toxique ». En conséquence, son discours est incarné sous la forme d'une objectivation d'elle-même, une réification qui ne laisse plus de place à la part de liberté du sujet, sa capacité à transcender cette permanence de soi par un véritable engagement, sorte de promesse, vers l'abstinence. En d'autres termes, Julie se retrouve figée dans cet en-soi qu'est le monde des déterminations et que Sartre a métaphoriquement nommé le visqueux: "Le visqueux s'appelle tantôt le passé, tantôt autrui, tantôt le monde » (Mounier, 1962, 57). Il y a bien une promesse qui peut lui permettre de décrocher et ainsi retrouver cette part de liberté constitutive du pour-soi : la promesse de retrouver un de ses fils, ainsi que nous le verrons infra. À l'inverse, pour David, la consommation est un choix, choix qu'il revendique et qu'il vit même sous le rapport d'une conquête vers une existence plus libre, ou à tout le moins libérée :

"C'est mon destin! Je l'ai choisi ce mode de vie et quelque part ça me conforte dans le fait que... Oui, c'est horrible mais ca me conforte dans mon identité de consommateur un peu... Enfin, consommateur un peu différent des autres consommateurs, complètement différent de ceux qui ne sont pas consommateurs, lancé dans une histoire de vie dont on pourrait faire parfois un roman. C'est gratifiant » (David).

Par le récit de sa vie, David construit un sens à son existence, celui d'un « aventurier maudit». Son vécu toxicomaniaque témoigne dès lors d'une forme d'auto-contrôle et de gestion qui n'est pas dominé par la souffrance mais par le plaisir :

"Tout le monde savait que j'étais "toxicophile" comme j'aime bien le dire. C'est pour moi un acte militant que d'en par- 
ler: "Regardez, moi je consomme de la drogue et je gère, voilà". Si j'employais le terme toxicomane, c'était plutôt dans une volonté plutôt de... Pas de choquer, mais d'exagérer un peu le terme. Parce que moi, je me suis rarement considéré comme un toxicomane, dans la mesure où, effectivement, j'ai eu des moments de toxicomanie, mais ces moments de toxicomanie, je m'en rendais relativement compte, et j'essayais de m'en extraire rapidement » (David).

Ainsi l'identité narrative de David rend compte d'un équilibre entre la permanence de son caractère et la capacité d'être à l'initiative de ses actes, de transcender ses déterminismes. Son rapport à soi s'inscrit dans la subjectivité, c'est-à-dire un pour-soi toujours en mouvement, jamais immobilisé :

«J'ai beau souvent me définir comme une espèce de merde humaine, j'ai un rapport à moi très particulier. Je pense qu'il y a peut-être des gens qui ont une image superficielle d'eux ou qui projettent une image superficielle bien plus valorisante, mais qui, en eux-mêmes, sont plus détruits. Tandis que moi, c'est le contraire. Quand je pense à moi superficiellement, je ne m'aime pas beaucoup, honnêtement. Après, quand je m'introspecte, je me vois des ressources quand même et ça, ça m'a bien servi » (David).

Tout en admettant qu'il est « atypique » et une «exception » dans sa trajectoire toxicomaniaque, sans trop pouvoir expliquer par quels moyens sa consommation « n'a jamais eu de véritable impact sur ma santé, jamais eu de véritable impact sur ma vie professionnelle, n'a jamais eu de véritable impact sur ma vie amoureuse », nous retrouvons dans son récit de vie le modèle de la promesse et de la fidélité qui, en projetant le soi vers l'autre, est constitutif de la dynamique de l'ipséité. Cette promesse l'engage auprès de ses parents, sa femme et ses enfants :
«Elle [Ma femme] avait quand même toujours plus peur que moi. Mais elle a bien vu quand même, au bout d'un an ou deux, que je savais effectivement fumer, déposer mon aluminium, aller chez mes parents, revenir, laisser mon aluminium ou ma boule deux jours, et puis refumer quand j'avais un peu de temps libre. Puis petit à petit avec les enfants venant c'est tout simplement d'autres activités, d'autres exigences, le fait que, à partir du moment que tu te mets des marqueurs, que tu ne peux pas fumer la veille parce que tu dois faire des trucs, à partir du moment où t'es quasiment tous les jours en train de faire un truc et que tu respectes tes marqueurs, tu fumes un peu moins » (David).

Saisir le rapport à soi par la mise en tension dialectique de l'idem et de l'ipse - de l'en-soi et du pour-soi - a pour mérite de montrer que l'apprentissage de la liberté, en tant que processus d'individuation et de subjectivation, s'effectue toujours dans un rapport constant avec l'altérité, à l'aune d'une dialectique intérieur-extérieur. C'est, en effet, que l'existant est fondamentalement communication. Il est même, pour Heidegger (1992), un Mitsein, un être-avec, de telle sorte que l'autre est constitutif du soi. De l'univers fusionnel avec la mère duquel l'enfant est séparé dès la naissance découle ainsi un travail de deuil et de renoncement à cette union symbiotique par l'apprentissage d'une nouvelle forme de complétude, celle de l'être séparé, singulier, autonome, «à nul autre pareil » (Adam, 2015, 60). C'est pourquoi, avant d'être un «je », l'enfant est un «tu » qui sous-tend un «nous», et dont les apprentissages sont conditionnés par les réactions de cet autrui qui le regarde. La conséquence est que la personne «n'existe que vers autrui, elle ne se connaît que par autrui, elle ne se trouve qu'en autrui » (Mounier, 1961, 36). C'est par cet autrui, et sa réintégration surmoïque, qu'elle fait l'apprentissage de sa liberté, en tant que personne séparée. 
Ainsi, si l'on veut saisir le sens que revêt la toxicomanie dans sa modalité pathologique, en tant que souffrance vécue sous la forme d'une aliénation comme c'est le cas de Julie, il convient dès lors de s'interroger sur ce rapport à l'autre.

\section{III - Le rapport à l'autre : manque à être et corps-sujet}

Emmanuel Mounier, en s'inscrivant dans la continuité des apports de la philosophie existentialiste, constate que « toutes les folies sont un échec du rapport avec autrui, alter devient alienus, je deviens, à mon tour, étranger à moi-même, aliéné. On pourrait presque dire que je n'existe que dans la mesure où j'existe pour autrui, et, à la limite : être, c'est aimer » $(1961,36)$. Bien loin d'une conception endogénéisante de la toxicomanie, une telle approche du pathologique a le mérite de poser le rapport dialectique, et les tensions qui peuvent en découler, entre l'intériorité et l'extériorité. C'est en étant animé par cette même réflexion d'une souffrance qui trouverait ses origines dans le socius, avant d'être intériorisée par le sujet, que Jacques Hochmann (1968) conçoit la sociopathie dans une approche relationnelle. Aussi, nous dit-il, « il n'y a pas de sociopathe à proprement parler, seulement des situations sociopathiques, des rencontres viciées entre un individu et un autre, un individu et un groupe et même, au maximum, un groupe et un autre groupe » (Hochmann, 1968, 196). C'est que, nous l'avons dit, l'Homme étant un être social, il ne se réalise pleinement que dans le dialogue avec l'altérité et c'est de ce dialogue que naît l'intuition de soi comme personne séparée et l'apprentissage de sa liberté-ipséité. La sociopathie incarne alors une maladie de la rencontre interhumaine qui, pour des raisons propres à chaque individu mais qualifiées de "sociopathogènes », entraîne un échec de la per- sonne «à répondre à l'appel légitime et à la demande du monde, et dans l'échec du monde à faire entendre cet appel » (Hochmann, 1968, 198). Cette structure morbide dialectique présente deux principales caractéristiques. D'une part, elle appartient au domaine décrit par Martin Buber (2012) du Je-Cela. L'individu rejeté est traité par le rejetant comme un objet, une chose, et non comme une personne. En conséquence, le rejeté enferme à son tour le rejetant dans un rôle défini et rigide, ce qui constitue, pour les deux pôles de la relation, une aliénation et une perte de l'identité personnelle: « Je ne suis plus qu'une chose pour l'autre mais parce que l'autre n'est plus qu'une chose pour moi, lui et moi devenons choses réellement 》 (Hochmann, 1968, 199). D'autre part, dans la continuité de la théorie de l'étiquetage (Becker, 1985), cette maladie de la rencontre est évolutive. Autrement dit, par son comportement, le rejeté provoque dans son entourage une série de réactions, celles-ci exagèrent la déviance, qui à son tour amplifie les réactions, selon le principe d'une causalité circulaire. La personne ainsi rejetée s'engage alors progressivement dans une carrière sociopathique qui peut s'exprimer de manière polymorphe, et notamment par la toxicomanie. En tant que maladie de la rencontre, une telle perspective situe la toxicomanie et lui donne sens, lorsqu'elle est pathologique, dans le champ des troubles du contact, ainsi que le cas de Julie l'illustre.

\section{A - Du manque originel à la passionnalisation de l'agir}

C'est dans cette perspective que Christophe Adam considère la toxicomanie comme un comportement relevant d'une perturbation du contact : « Une pratique dans et par laquelle le sujet en état de manque cherche à s'adjoindre un complément pour éprouver un sentiment 
de plénitude, sur le mode du contact fusionnel. [...] Le sujet se complète ainsi de ce dont il est manquant - du moins se vit comme tel » (Adam, 2015, 72). Aussi, le «manque à être » (Le Breton, 2012, 55 ) est originel - presque ontologique chez Julie. Il est né dans le rapport à l'autre, plus particulièrement à sa mère, à l'aune d'un manque affectif :

«Elle ne m'a pas prise près d'elle, elle m'a avoué : “Je ne t'ai pas prise, j'ai demandé qu'on te laisse en néo-nat, tout le long de mon séjour en maternité, parce qu'on est restées huit jours. Et je ne voulais pas m'occuper de toi, parce que je ne te voulais pas. Je ne te voulais pas, je ne voulais pas d'une fille, d'une pisseuse, chieuse, comme tu l'es encore maintenant" » (Julie).

«Ma mère m'a maltraitée toute ma vie, vulgairement, en paroles. J'ai été une enfant traumatisée par les paroles méchantes, blessantes, de la part de ma mère. Elle disait: "Vous voyez ça ? - en me montrant - C'est la plus grosse connerie que j'ai faite de ma vie, mettre ça au monde !" » (Julie).

« Vous voyez ça ? » Cette citation exemplifie magistralement la relation Je-Cela caractéristique du rapport entre Julie et sa mère. Le «ça» appartient au domaine du non-être, du vide, et donc du manque. Julie est chosifiée par sa mère dès son enfance, elle ne peut donc faire l'apprentissage de la séparation, puis de la complétude retrouvée par la présence de l'autre en soi, comme instance surmoïque. Elle se retrouve seule, livrée à elle-même et à ses motions pulsionnelles, siège du «ça ». C'est en ce sens qu'on peut, dans son cas, parler de passionnalisation de l'agir, "soit la transformation de l'usage en abus » comme "voie de détour, une façon de couper court à ce traumatisme de la perte et de la séparation. Le sujet se soustrait au deuil à faire grâce à l'addition » (Adam,
2015, 74) par la consommation de produits. Celle-ci apparaît alors comme une béquille permettant de pallier cette angoisse d'abandon. Une "médicalisation de la souffrance existentielle » (Le Breton, 2012, 56) qui a lieu très tôt dans le vécu de Julie; dès l'âge de 10 ans, elle va chercher à combler son manque en sniffant les Valium de sa mère. À 15 ans, elle rencontrera l'héroïne dans le rapport à l'autre; c'est en l'autre qu'elle voit le reflet de son manque et l'occasion de calmer son angoisse d'abandon :

« J'ai commencé à prendre de l'héroïne, j'avais 15 ans. J'ai commencé avec mon meilleur ami, à l'école. C'était un orphelin, et moi, je m'y ressentais un peu, je me sentais bien avec lui parce qu'il vivait sans parents. Je me sentais bien en lui parce que, lui, il manquait d'amour de ses parents, et moi je manquais totalement d'amour de mes parents aussi finalement, dans un autre contexte mais, on avait le même manque. C'est là que j'ai appris à prendre l'héro, c'est là que j'ai appris tout, avec lui » (Julie).

David n'exprime pas de manque originel, il verbalise un amour vécu à travers la douceur de sa mère et l'éducation tyrannique de son père :

« J'ai eu de la chance avec mes parents, avec la force qu'ils ont pu me donner, avec l'amour que... Tu vois ? Ce n'est absolument pas anodin. Moi, quand je vois tous mes potes qui sont morts d'overdose, suicide, accident, à cause des consommations, je regarde leur cadre familial, honnêtement, je n'en vois pas un qui avait la chance d'avoir le même que le mien. Alors que j'ai un père tyran, presque tyran. Il y avait quand même, il y avait tellement d'amour chez moi. Je pense à mes deux-trois meilleurs amis qui sont morts d'overdose ou de suicide, ils avaient tous des parents absents, des parents morts, des parents dépressifs » (David). 
David ne manifeste pas un manque qu'il chercherait à combler à travers sa consommation. Son récit de vie témoigne d'un apprentissage de la séparation qui s'est effectué à l'aune d'une relation Je-Tu. Cet amour de ses parents lui a ainsi permis de les réintégrer comme instances surmoïques, comme nous allons plus amplement le voir infra.

\section{B - Gestion du manque phy- sique dans le rapport à l'autre}

Le manque faisant partie de Julie, celui provoqué par la prise de produit n'a jamais été exprimé pendant l'entretien. Un manque existentiel est là, la souffrance occasionnée par le produit est alors tapie dans l'ombre de l'être qui en souffre et qui en a toujours souffert. David exprime à plusieurs reprises le manque physique et la peur de ce manque. Le manque est alors objectivé à travers la peur, alors que l'angoisse - fondamentalement sans objet - habite Julie. Le manque se nomme chez David, il se vit enfoui en Julie. Il a réussi à apprivoiser «un ennemi nommé et visible » alors qu'elle a été confrontée à " l'insaisissable horreur qui se cache au cœur de nous-même » (Mounier, 1962, 53).

C'est la peur, et non l'angoisse, qui habite David. Cette peur n'est pas subie mais investie dans le rapport au produit :

"J'ai très peur de devenir toxicomane. [C'est] sans doute la douleur, la douleur physique. J'avais peur du manque, ça m'a toujours fait peur. Mais finalement, est-ce que [le fait] d'avoir peur, ce n'est peut-être pas plus utile que d'avoir de la volonté ? Tu vois ce que je veux dire? Alors, ok, je peux me définir comme un couillon. Mais le fait d'avoir peur d'avoir mal, est-ce que ça ne m'a pas plus servi qu'être une espèce de... En disant : "Moi je vais y arriver, je suis sûr de moi, j'ai de la volonté !". Parce qu'avec de la came, à la fin, tu meurs quand tu joues ce jeu-là. Enfin, tu perds, je pense, tu vois ? Tandis que moi, j'avais beaucoup de respect, enfin, de respect entre guillemets, pour le produit. Beaucoup de craintes, c'est ça que je veux dire et pas beaucoup de respect pour mes capacités. Et je pense que c'est quelque part ça aussi, tu vois, les forces peuvent être des faiblesses » (David).

Dans leur rapport au produit, l'angoisse renvoie, chez Julie, à la polarité passive alors que la peur renvoie, chez David, à la polarité active. De cette polarité naissent des capacités de gestion de la consommation très différentes. Julie subit le produit alors que David présente des aptitudes à la maîtrise de sa consommation à travers la gestion du manque :

«Parce que, à cette époque-là [lors de ma première overdose], je me disais que jamais j'arrêterai. J'aimais trop! Mais c'est comme maintenant, la drogue, j'ai arrêté parce que j'ai eu des problèmes ici [à la maison d'accueil], mais je sais que tous les $X$ mois... L'été passé, j'ai eu une rechute aussi! Six mois après, j'ai eu une rechute! Dans six mois, j'aurai une rechute! J'ai des rechutes de temps en temps. Je n'y crois plus! Il n'y a aucune cure, même là, ils me disent: "Tu vas aller faire six mois à [l'hôpital]". Je peux te mettre ma main à couper qu'après ça, il y aura encore des rechutes $\gg$ (Julie).

«Moi, comment je gérais mes manques? Pas de substances pour diminuer l'impact. Et le lendemain, toujours aller bosser, ne jamais me mettre un certificat médical à cause d'un problème de toxicomanie. Donc, encore une fois, qu'est-ce que je faisais ? J'essayais à nouveau de calculer mes consomma- 
tions quitte à refumer une micro-tache ${ }^{3}$. Arrêter de fumer par exemple le mardi, donc fumer une micro-tache (il appuie sur le "micro") le mercredi matin par exemple, parce que je sais que sinon je vais être en manque. Mais je sais que grâce à cette micro-tache, je vais être en manque à minuit. Et là à minuit, je me serai endormi et donc le manque, il va passer. Et donc, je vais me réveiller avec sans doute encore plein de douleur comme si j'étais grippé, trempé parce que j'ai transpiré toute la nuit, peut-être un petit mal de tête, mais j'aurai passé le gros du manque. Parce qu'encore une fois, moi je ne suis jamais arrivé à un stade où le manque me réveillait, jamais. Alors que des amis, oui. S'ils faisaient comme moi, entre parenthèses, ils n'y arrivaient pas parce qu'ils étaient trop loin » (David).

Cette capacité ou non à gérer le manque et la consommation passe par une dé-corporation, c'est-à-dire une capacité à la réflexivité physique. Cette prise de distance par rapport au corps physique se nourrit de la peur de la douleur, mais aussi du corps extérieur, le corps social. Ce corps est mort chez Julie. Non seulement elle porte les stigmates psychiques du rejet maternel, mais en réaction, elle a rejeté ce corps qui ne s'est jamais réellement greffé à elle, qui tout au long de sa trajectoire lui est resté étranger.

«[À ses parents]: "C̣a fait depuis mes 15 ans que je prends de la drogue, et vous êtes tellement attentifs envers moi que vous vous êtes rendus compte de rien! Ce n'est pas ça des parents !" Et alors, j'ai dit: "Moi, je quitte la maison, je quitte la famille, je quitte tout et tout le monde, et je me casse!" »(Julie).

« Je n'ai personne dans ma vie. Il n'y a pas plus seule que moi. Je n'ai aucune amie. Mes parents ? Ma mère est morte l'année passée. Mon père, on a coupé les ponts. Mes enfants, je ne les vois pas, le grand ne veut plus me voir. Les amis, j'ai beau chercher, je n'en ai pas » (Julie).

David, quant à lui, puise dans le corps social - ses parents - les ressources lui permettant de maîtriser sa consommation et ses manques physiques :

« Le contrôle avec certains indicateurs de contrôle, voilà. Et ça, je me suis toujours tenu à ces indicateurs de contrôle. Et ma principale motivation étant ma peur de la douleur, du malheur en général, de la douleur physique aussi et mon rapport aux parents » (David).

La souffrance du corps physique est alors endurée sans traitement de substitution et maîtrisée par des stratégies de gestion pour éviter la souffrance du corps social :

" Je crois que je préfère mourir que le décevoir [mon père]. Enfin, souffrir le mal de manque plutôt que le décevoir. Ca c'est une certitude! Ce qui fait que je m'arrangeais toujours, même quand je n'étais pas étudiant, et que j'étais jeune travailleur, je rentrais une fois toutes les deux semaines chez moi, et bien, il était impensable que mes parents découvrent que je fume » (David).

\section{C - Corps-objet / corps-sujet}

Cette interaction entre corps physique et corps social est rendue possible car, dans le cas de David, son corps social est vivant et son corps physique, incarné, est éprouvé en tant qu'espace de communication entre intériorité et extériorité. Le vécu de David témoigne d'un corps qui n'a pas été chosifié. Il s'agit d'un corps investi, écouté et respecté : un corps-sujet. 
David est à l'écoute de «son » corps et cela conditionne son rapport à la drogue. Contrairement à Julie, il ne consomme le produit par intraveineuse qu'à deux reprises. Le fait de se trouer lui semble trop violent, «l'effraction cutanée de l'injection intraveineuse » (Hachet, 2005, 67) constitue pour lui une agression d'un corps qu'il respecte.

« C'est la même chose qui m'a empêché de me shooter hein, c'est la douleur et la peur de l'aiguille. [Je me suis shooté] deux fois parce que j'avais envie d'y goûter quoi. Ce n'est même pas moi qui ai fait les injections les deux fois. Donc, je me suis dit: "Voilà, oui, c'est chouette, c'est vraiment très chouette, mais non. Ce n'est pas pour moi quoi, c'est vraiment trop violent. Rien que la pensée de me trouer". Quelque part, j'ai un respect, un rapport à mon corps que, je pense, il n'y a pas chez tout le monde » (David).

Au moment de l'entretien, David n'a plus consommé depuis plusieurs mois. Cette sortie de la toxicomanie a été possible grâce à la rencontre du corps-sujet physique, lorsqu'il fait un infarctus, et de supports sociétaux (Castel, 1994) membres de son corps social.

«Elle [ma consommation] a diminué à un moment [où] je ne pouvais, de toute façon, me marier que si j'étais en phase de changement, dans un sens large. Sinon, je n'aurais pas voulu me marier. Et qu'une fois marié, ce changement-là a accéléré les autres changements. J'étais heureux en couple, j'avais des enfants. Oui une petite tache, je ne dis pas non, mais pas deux fois par semaine hein gros! C'est bon quoi, une petite tache de temps en temps, mais je n'ai pas envie d'être défoncé, de piquer du nez quand mon enfant vient me demander de lui raconter Homère, l'Iliade et l'Odyssée, tu vois ? Et donc ça, ça m'a préparé à mon infarct, c'est-à-dire, au moment où tu te dis: "Là, je dois peutêtre vraiment, vraiment, vraiment diminuer !". Et ça a été vraiment facile pour moi de répondre à ce stimuli qu'est l'infarctus parce que j'étais déjà dans une période où... Et donc là, j'ai vraiment arrêté pendant plusieurs mois, puis j'ai recommencé à fumer de façon, je ne dirais pas comme avant, mais un peu plus quand même. Et puis petit à petit, ça s'est simplement délité » (David).

A contrario, Julie dispose d'un corps-objet. Nous avons vu comment elle avait été, dès sa naissance, chosifiée par sa mère. Le reste de sa trajectoire de vie ne fait qu'amplifier cette chosification d'un corps volé par le regard et les intrusions de l'autre, de telle sorte qu'elle ne peut plus se l'approprier.

« Je n'ai jamais été belle, jamais Isanglots)! Et on se foutait de ma gueule tout le temps. Il y a de quoi, je sais, mais c'était méchant. Ils se foutaient tout le temps de ma gueule, je me suis toujours dévalorisée » (Julie).

Cette dévalorisation symbolique intériorisée se concrétise à travers le vécu de Julie. Elle subit la violence du monde de la drogue sur son corps. Celui-ci nous est alors montré pendant l'entretien, il est corps-témoin ou, plutôt, corpspreuve des supplices endurés.

«Regarde, aussi à cause d'un mec, cinquante-huit agrafes. Il m'a poussé d'un mur de six mètres de haut. Je suis tombée en arrière sur le macadam. Là, la clavicule a explosé. L'os sortait hein! T'avais la clavicule qui arrivait. Et tu sais ce qu'il a fait ? Il m'a laissée dix jours dans mon divan à crever avec l'os qui ressortait! Au bout de dix jours, il m'a emmenée à l'hôpital. Le docteur regarde dans les radios, je l'ai entendu crier: "Mais comment cette femme n'est pas morte de douleur ?" » (Julie). 
Julie parle souvent de son corps en employant, non des déterminants possessifs, mais des articles définis: " la clavicule a explosé », «l'os sortait», «t'avais la clavicule qui arrivait », « l'os qui ressortait». Si «l'homme est à la fois un corps-sujet et un corps-objet, un corps que l'on "a" et un corps que l'on "est" » (Marzano, 2009, 7), Julie ne semble pas être un corps mais avoir un corps qu'elle montre et qui est montré, mais qui n'est pas vécu.

Le paroxysme de la conception du corps comme objet est atteint dans le vécu de Julie lorsqu'une partie de son corps est sortie de son enveloppe et observée hors-de-soi à la suite d'un des viols qu'elle a subis :

« J'ai vécu quatre viols. Le premier, j'ai eu vingt-sept points de sutures à l'intérieur. Ils m'ont rentré des bouteilles avec le goulot cassé. Il y avait du sang sur tout le lit. J'avais du sang collé dans les cheveux. J'étais faible comme une morte vivante, j'ai vomi de la mousse blanche, j'ai vomi en voyant ce que j'ai vu à côté : les bouteilles, avec ce qu'il y avait eu dessus, là » (Julie).

Ainsi, si c'est dans le rapport à l'autre que s'élabore cette souffrance constitutive du pathologique, celle-ci est par la suite réintériorisée par le sujet, tant dans ses manifestations psychiques que somatiques. À l'inverse, que le sujet soit dans la polarité passive ou active, qu'il investisse ou n'investisse pas un corps-sujet, il est offert au monde et doit se projeter en celui-ci, dans un mouvement contraire allant de l'intérieur vers l'extérieur. Cette projection n'est jamais prédéterminée, mais elle est le fruit d'une réinterprétation constante de soi dans le monde, à travers un équilibre fragile entre la vie et la mort.

\section{IV - Le rapport au monde au prisme de la polarité vie-mort}

Georges Canguilhem conçoit la vie comme une activité normative, à savoir le fait que «la vie n'est pas indifférente aux conditions dans lesquelles elle est possible, que la vie est polarité et par là même position inconsciente de valeur » (Canguilhem, 1975, 77). Cette normativité biologique relève, selon l'auteur, d'un effort spontané du vivant pour lutter contre tout ce qui peut entraver son maintien et son développement, ces derniers étant pris pour normes, c'est-àdire comme valeur positive - type idéal de la polarité dynamique de la vie 4 . Il est donc inscrit en germe dans le bios de retenir dans son environnement ce qui lui permet de tendre vers le pôle positif de la vie et de rejeter tout ce qui peut l'entraîner vers le pôle négatif - avec comme valeur absolue son extinction de telle sorte que "vivre c'est, même chez une amibe, préférer et exclure » (Canguilhem, 1975, 84). En ce sens, la thérapeutique, tour à tour activité religieuse, magique, puis scientifique, apparaît pour l'auteur comme une technique humaine visant à prolonger ces impulsions vitales par une connaissance systématique de la maladie. Pour ce faire, « le vivant humain qualifie lui-même comme pathologiques, donc comme devant être évités ou corrigés, certains états ou comportements appréhendés, relativement à la polarité dynamique de la vie, sous forme de valeur négative » (Canguilhem, 1975, 77).

On comprend, dès lors, en quoi la toxicomanie peut être envisagée, en rapport à la polarité dynamique de la vie, comme un type spécifique relevant du pathologique et le toxicomane comme une figure d'exclusion. Elle constitue, d'une part, une intoxication de l'organisme pouvant entraver les fonctions vitales

(4) En témoigne la réaction de défense " naturelle » de tout organisme contre l'inoculation pathologique par la création d'anticorps, et ce afin d'un retour à un type idéal qu'est la santé. 
de la personne. Mais surtout, et d'autre part, elle apparaît comme une limitation de l'être humain à une condition unique et invariable - « utilisation répétée du produit conduisant à l'incapacité de remplir des obligations majeures, au travail, à l'école ou à la maison » (American Psychiatric Association, 2013, 541) - par référence «à l'idéal normal humain qui est l'adaptation possible et voulue à toutes les conditions imaginables » (Canguilhem, 1975, 87).

Une telle conception de la conduite toxicomaniaque la réduirait ainsi, en regard à cette polarité vie-mort, à une signification unique d'autodestruction, en tant que conduite visant l'anéantissement du sujet. Or, les récits de vie de Julie et David témoignent d'une trajectoire toxicomaniaque prenant un sens et une place dans leur vécu bien plus complexe que la seule fonction autodestructrice. Pour envisager cette complexité, il nous semble particulièrement heuristique de faire dialoguer le principe de normativité biologique tel qu'énoncé par Canguilhem et le concept d'être-au-monde mis en avant par Heidegger (1992). Lorsque Canguilhem formule l'idée «que la vie est polarité et par là même position inconsciente de valeur » $(1975,77)$, il prolonge ainsi la thèse selon laquelle l'être-au-monde n'est pas qu'un simple réceptacle - un « être-jeté »- des conditions extérieures objectives. L'existant, s'il est toujours situé dans un environnement, et « dans l'impossibilité de prendre sur le monde une autre perspective que celle que lui donne sa situation » (Mounier, 1962, 93), n'en reste pas moins un être en mouvement, engagé dans son monde en vue de lui donner un sens et le transformer. Aussi, la toxicomanie, tant dans son versant pathologique que non pathologique, apparaît comme une manière pour le sujet de s'adapter à un environnement qui peut être pathogène, en vue d'introduire un nouveau rapport au monde non dirigé uniquement vers la mort mais aussi vers la vie. Elle est, en ce sens, normative, et peut relever de différents registres de signification eu égard à cette polarité dynamique de la vie.

\section{A - La mort...}

Si « supporter la vie reste bien le premier devoir de tous les vivants » (Freud, 2012,82 ), se rapprocher volontairement de la mort est un geste d'exclusion. À cet égard, le toxicomane semble avoir opéré ce basculement puisqu'il est "suspecté travailler à sa propre destruction » (Lambrette, 2008, 19) : il se shoote, il s'inflige des coups dans la violence de la pénétration de son corps (Desfourneaux, 2008). Tout élan vital lui est alors confisqué et, dans l'imaginaire collectif, «le drogué heureux n'existe pas » Olievenstein, cité par Desfourneaux, 2008, 210).

Le vécu de Julie semble, a priori, traduire cette auto-exclusion :

«Pour soigner quelqu'un qui est blessé, je fais tout. Mais quand c'est pour qu'on vienne me soigner moi... Mon hépatite $C$, ça fait quinze ans que je l'ai, on m'a proposé au moins 25 fois le traitement. "Plus tard, plus tard, plus tard...". De toute façon, je n'ai personne dans ma vie (long silence). Et ça serait un miracle si je rencontrais l'amour. J'ai décidé de ne plus jamais croire en l'amour » (Julie).

Au cours de l'entretien, Julie nous montre le tatouage inscrit sur sa main: elle a effacé le « 0 » de «LOVE »; elle a effacé l'amour de son corps, objet-témoin de sa désillusion vis-à-vis de cette valeur qui rattache à la vie. Le refus de soin qu'elle exprime peut d'ailleurs être considéré comme une conduite suicidaire. Elle a ainsi, à maintes reprises, exprimé une envie de mourir, parfois sous la forme d'une digression comme pour en souligner l'évidence : 
«Tu vois que les baffes me sont restées, et elles me resteront jusqu'à ma mort, et... Mais en parlant de mort, je viens de penser à quelque chose parce qu'en ce moment, je suis tellement mal dans ma peau que j'ai envie de mourir » (Julie).

Elle est d'ailleurs déjà passée à l'acte avec une tentative de suicide par overdose pour laquelle elle nous exprime son regret de ne pas avoir réussi à mettre un terme à son existence :

«Mon overdose, je l'avais faite en allant chercher mes ordonnances. J'étais à la pharmacie, ici, et sur le chemin dans le tram, j'ai avalé mes quatorze gélules, plus une plaquette de Bromazepam douze milligrammes. J'ai tout avalé. Ils sont venus, ils ont senti mon pouls, mon pouls ne battait plus, alors ils ont appelé l'ambulance. Ils ont essayé de me réanimer. Puis après, ils ont réussi à me réanimer mais je dis, et je dis bien, face-à-face, je te regarde dans les yeux: "Malheureusement!" Je me dis: “Autant en rester là, finir de souffrir". Parce que moi, je souffre énormément » (Julie).

Julie donne un sens à son suicide : se libérer de sa souffrance et libérer son enfant de l'incertitude :

«Malgré que quand je dis ça [que je veux mourir], on me dit: "Et ton fils, même s'il ne te réclame pas maintenant, à six ans et demi, si dans, même dans dix ans, s'il te réclame et qu'on lui apprend que t'es morte d'une overdose, quand il avait 6 ans, comment il va réagir ?". Mais, je dis : “Au moins, il sera fixé, il aura sa famille. Il a déjà sa famille, je ne saurai jamais lui apporter ce qu'ils lui apportent". Alors je me dis autant en rester là, finir de souffrir, parce que moi je souffre énormément, je suis en énorme dépression » (Julie).

\section{B - ... comme appel jeté au monde}

Les tentatives de suicide «sont des conduites complexes, souvent ambiguës, qui ne sauraient être définies par une finalité univoque d'auto-destruction » (Vauterin, 1963, 160). S'arrêter à l'envie de mourir de Julie, c'est la condamner à l'exclusion, la laisser du côté des morts. Or, si «l'homme est condamné au sens, il convient de rechercher, dans toute conduite suicidaire, ce sens, cette intentionnalité qui ne sauraient être la mort seule, négation de sens, désengagement extrême. Cette intentionnalité n'est pas le fait d'une conscience suicidaire artificiellement isolée, mais d'une conscience engagée dans le monde et dans la relation à autrui » (Vauterin, 1963, 180). Lorsque Julie énonce son envie de mourir, elle fixe notre regard, attend une (rélaction :

«Et je vais le faire! Je vais faire ces papiers-là [qui énoncent un refus de réanimation en cas d'arrêt cardiaque] ! Et le jour où j'aurai l'occasion, j'aurai une overdose et je... Ce n'est pas un problème que je dise ça ?» (Julie).

La conduite suicidaire de Julie a une fonction d'appel à l'autre, au monde. Happée par le néant, sa conduite suicidaire est un social releaser (Vauterin, 1963, 183), pour que l'autre puisse la raccrocher à la vie. Par l'interrogation «ce n'est pas un problème que je dise ça?», Julie suscite une réponse du chercheur. C'est dans cette même logique qu'elle a annoncé sa toxicomanie à ses parents. Elle recherche désespérément une réponse sociale à sa souffrance. D'ailleurs, lorsqu'elle a tenté de se suicider, elle a été vers l'autre, dans un centre d'aide aux toxicomanes. Dans le cas de Julie, il semble évident que l'intention suicidaire est « un mouvement de l'être [...] tourné vers autrui 
[...] » (Vauterin, 1963, 161). Si sa prise de produit a pour fonction de combler le vide creusé dans l'absence de l'autre, ses intentions suicidaires ont - également - pour fonction de la rapprocher de la vie. Julie s'en prend à son corps «pour prendre la vie à bras le corps» (Desfourneaux, 2008, 198). L'extrait suivant illustre d'ailleurs qu'elle est un être tourné vers la vie, dans l'ombre de la mort :

«J'ai été au SPJ [service de la protection de la jeunesse], et là ils m'ont dit que du bien [à propos de mon fils placé]. Et en revenant de cette réunion, j'ai dit à tout le monde : "Fini de vouloir mourir ! Je veux vivre! J'ai rattrapé espoir pour mon fils !". Et ça revient... mes envies reviennent. Alors, je dois vivre parce qu'il y a espoir vis-à-vis de mon fils. Mais je vis tellement mal que je ne sais pas quoi faire. Peut-être que cette augmentation de méthadone, peut-être que ça va me faire du bien » (Julie).

\section{C - L'ordalie comme gestion de la tension entre pulsion de mort et pulsion de vie}

Dans le cas de David, également, la polarité vie-mort ne saurait être réduite à un de ses extrêmes. David joue et se joue de la mort, il l'a côtoyée à travers les suicides et les overdoses de ses partenaires de défonce :

"C'est terrible ce que je vais te dire: [la mort de mes amis, je l'ai vécue] à la fois très, très mal parce que c'est d'une violence inouïe, et à la fois pas bien, mais à la fois où tu te dis: "Tiens, moi j'y échappe". Putain, c'est hallucinant. Et en plus se conforter, dans le sens où tu vis un destin un peu exceptionnel même s'il est horriblement malheureux. Et que c'est peut-être mieux de vivre quelque chose de terriblement malheureux que de bêtement heureux. Tu vois ce que je veux dire ? » (David).

Ses épisodes «violents » et «malheureux », au lieu de l'éloigner du monde de la drogue, ont participé à la construction d'une image de soi qui correspond aux identifications de David : être un perdant magnifique, un maudit aventurier :

«Moi, je voulais être une petite frappe. Je m'étais déjà totalement identifié aux blousons noirs de la rue, à 12 ans. Donc, je ne rêvais pas d'être Al Pacino ou le grand truand. Moi [ce que] je rêvais d'être, c'est cette espèce de perdant magnifique » (David).

«Il y avait deux choses : le plaisir lié à la consommation et le plaisir lié à l'image liée à la consommation, à l'image d'un aventurier maudit et héroïque qui a affronté beaucoup de... Torturé à l'intérieur alors qu'il y avait aucune raison d'être torturé » (David).

Pour atteindre ses aspirations, David a eu besoin de la mort; dans son cas, «la vie s'appauvrit, elle perd de son intérêt, dès l'instant où dans les jeux de la vie, il n'est plus possible de risquer la mise suprême, c'est-à-dire la vie elle-même » (Freud, 2012, 55). Aussi, sa consommation revêt une fonction ordalique, comme «façon de s'en remettre à l'Autre, d'en appeler à une puissance tutélaire, à un jugement des dieux pouvant témoigner de notre valeur » (Adam, 2015, 75 ; Le Breton, 2012). Il s'agit alors de prendre tous les risques, parier la mise suprême et s'en remettre à la mort pour se sentir exister, et ainsi rendre le vivant plus vivant.

Julie manifeste également un rapport ordalique au produit. Cependant, celui-ci revêt une autre fonction. Plutôt que de lui donner le sentiment d'une existence « exceptionnelle », il est une manière de gérer la tension entre pulsion de mort et 
pulsion de vie, en s'en remettant ainsi à une pensée magique toute désignée afin de trancher quant au sens que Julie ne parvient plus à donner à sa vie (Vauterin, 1963, 183-184).

« Et il est arrivé que je shoote gramme par gramme. Alors, quand je raconte ça à certains toxicomanes, ils me disent: "Ouais, ça ce n'est pas possible, tu serais morte". Ben non, apparemment j'ai une étoile au-dessus de la tête. J'ai fait quatre ${ }^{5}$ overdoses dans ma vie, et je ne sais pas pourquoi, on ne me veut pas au-dessus » (Julie).

« Je commence à croire un tout petit peu en Dieu. Parce que sinon je serais morte, s'il n'y avait pas un bon Dieu » (Julie).

Alors que dans le cas de Julie « la toxicomanie est une expérience de mort et de renaissance, [...] par le jeu d'une ordalie toujours renouvelée » (Le Breton, 2012, 62), dans le cas de David, se joue le maintien du côté des vivants, loin du modèle «marginalité/héroïne/ exclusion » (Faugeron et Kokoreff, 2002, 19). Mais ce rapport ordalique ne fait, selon David, pas uniquement appel à une volonté divine ou à la chance, il est conscient qu'entre lui et l'exclusion, il y a un corps social et ses ressources.

« J'ai de la chance, mais [les] circonstances étaient pour moi hyper [positives]. L'environnement, je ne vois que ça, parce que le produit était le même que tous mes potes. Je ne crois pas être Superman à part, peut-être, deuxtrois qualités en plus. J'avais peut-être deux-trois trucs en plus, mais je crois que c'est surtout ces putains de circonstances qui étaient super bien. On ne m'a jamais mis le doigt dessus. On ne m'a jamais critiqué. On m'a toujours laissé vivre ça cool avec toutes les ressources, les renforts, tous les back up que j'avais. C'était super ! » (David).
L'ordalie incarne donc cet espace intermédiaire, lieu d'affrontement entre la vie et la mort. Si son recours relève de registres de significations différents pour Julie et David, il n'en reste pas moins que pour tous deux, vie et mort s'alimentent dans un rapport dialectique. Néanmoins, dans ce continuum allant de la mort à la vie, David témoigne d'une dernière fonction de la prise de produit qui le raccroche, cette fois-ci, uniquement du côté de la vie.

\section{D - Une prise sur la vie}

David rompt radicalement avec l'image de la toxicomanie comme désir d'auto-destruction. Au contraire, il exprime, tout au long de l'entretien, son épanouissement dans la drogue :

« On vivait comme ça, des consommations, des moments de consommation relativement fortes, ininterrompues pendant deux-trois semaines. Et ça, c'était le bonheur, putain quoi! C'était le bonheur » (David).

«Alors, l'héroïne, une grosse partie ça été que du bonheur, que du plaisir, que de la récréation » (David).

Rappelons-nous que David se définit comme un «toxicophile », revendiquant par-là que sa consommation d'héroïne n'a jamais été une souffrance mais au contraire un intermédiaire lui permettant de goûter et d'aimer la vie plus intensément. Le contact avec le produit agit dès lors comme un amplificateur de sensations, permettant ainsi $d^{\prime}$ ' hyper-esthésier » son rapport au monde (Adam, 2015, 64). Ce contact a partie liée avec l'ambiance et l'humeur et constitue une mise en mouvement de la vie sous une forme hédonistique.

Néanmoins, au-delà du simple plaisir, la prise de produit permet aussi de 
répondre aux exigences d'une société de performance et ainsi s'adapter à un environnement potentiellement créateur de souffrance en s'adjoignant un « améliorant » permettant d'y faire face :

« Je ne saurai pas t'expliquer le stress! Honnêtement, c'est intransmissible le stress qu'on nous fait subir parfois dans certains trucs de boulot. Et donc effectivement, de façon ponctuelle, il m'est arrivé de consommer de l'héro sur mon lieu de travail pour faire tomber le stress, clairement. Et je travaillais mieux. La consommation en elle-même, ça me rendait plus efficace. Plus empathique, moins stressé, plus détaché, oui, franchement $\gg$ (David) ${ }^{6}$.

L'usage de produits psychoactifs peut donc relever d'un effort dynamique d'adaptation et d'engagement dans le monde, en rapport à cette polarité viemort, et à rebours de toute conception psychiatrique qui figerait le toxicomane dans les déterminismes du produit sans prise en compte de l'environnement dans lequel il s'insère. La proximité avec la mort agit alors comme élan vital, comme appel jeté à l'autre ou comme porte ouverte sur un destin extraordinaire, et permet de déconstruire la toxicomanie dans sa face univoque d'autodestruction pour, a contrario, l'envisager à certains égards comme une prise sur la vie.

\section{Conclusion}

Au travers des récits de vie de Julie et David s'ébauchent deux vécus toxico- maniaques que l'on pourrait qualifier de bipolaires. Une telle mise en dialectique permet dès lors de rendre compte à quel point l'étiquette accolée au toxicomane produit une identité figée et réduite au seul champ du pathologique, manquant ainsi toute la singularité et la complexité des rapports qui peuvent se nouer entre le sujet et son produit : «Plusieurs reflets d'une même identité semblent ainsi coexister et concourir à l'éclatement du profil de l'usager de drogues » (das Neves Ribeiro, 2018, 578). Il y a bien une modalité pathologique de la toxicomanie, lorsque le rapport au produit s'élabore au prisme d'un vécu de souffrance. Mais celle-ci ne peut être désignée comme telle que par celui qui l'éprouve et non en fonction d'un idéal de fonctionnement « normal » que la société se donne et que la science légitime après-coup. Aussi, l'addiction ne peut être mesurée qu'au seul regard de critères quantitatifs. De même, elle ne peut être essentialisée aux seules caractéristiques du produit ingéré. Nous l'avons vu, la toxicomanie prend toujours sens dans un certain rapport à soi, aux autres et au monde. Et si l'utilisation du produit peut transformer l'horizon de ces rapports, elle ne répond pas seulement à un appel du néant, mais reste une manière de s'adapter à un environnement souvent pathogène en vue de continuer à maintenir un certain rapport à la vie, et ce par l'introduction de nouvelles normes permettant un équilibre précaire. C'est en ce sens que, si l'on veut véritablement comprendre le sens que revêt la toxicomanie dans la singularité d'une existence, il est nécessaire de toujours l'envisager selon une approche globale qui resitue l'insertion du produit dans les interactions dynamiques entre corps social, corps psychique et corps physique.

(6) L'instrumentalisation du produit à des fins professionnelles n'a eu lieu, dans la trajectoire de David, qu'à de rares occasions. Si une prise contrôlée d'héroïne semble améliorer ses performances face à des conditions extrêmes il est conscient du poids du contrôle social et de ses possibles conséquences sur sa vie professionnelle. Ainsi, celui-ci protège cette sphère d'activité, car le travail permet à David de " se différencier du "toxicomane", de celui qui ne peut pas avoir un bon travail puisqu'il organise sa vie autour des produits et de la marginalité qui les entoure. [...] Utiliser des produits en maintenant son intégration sociale par le travail [permet ainsi à David] d'échapper à l'image défaillante du "drogué" [...] » (Fontaine, 2006, 68). David illustre ainsi l'interdépendance des identités sociales et personnelles. En ayant une estime favorable de lui-même, David est d'autant plus habilité à contrôler et manier les informations qu'il partage avec son corps social. Cette résistance au stigmate lui permet de continuer d'investir et d'alimenter son identité sociale, et maintenir son intégration (Doyen et Das Neves Ribeiro, 2019). 
Selon une approche phénoménologique, nous sommes ainsi partis du vécu des interrogés et du sens qu'ils donnent à leur consommation pour plonger leur récit dans « les bains révélateurs des théories et concepts mobilisés » (Molitor, 2019, 6), et ce afin de montrer que rapport à soi, à l'autre et au monde sont intimement liés. Ainsi, c'est dans l'interaction entre rapport à soi et rapport à l'autre que naît la liberté et donc la faculté de prendre distance par rapport à un idem qui risque d'engluer le sujet dans l'identité toxicomaniaque. Cette liberté permet à David de s'éprouver comme un corps-sujet incarné en communication avec un corps social lui permettant de gérer sa consommation et ses manques physiques. Julie, a contrario, a été chosifiée, figée dans le rapport à l'autre. Son corps-objet, vidé de son équilibre, nécessite alors un produit-béquille pour se projeter au monde. Cette projection est nourrie intérieurement et extérieurement, et peut s'appréhender à travers la polarité vie-mort. Cette approche polarisante est cependant un outil d'analyse : ni Julie ni David n'investissent exclusivement l'une des deux. Le suicide, dans son désir et ses tentatives, permet à Julie de se raccrocher à l'autre et, donc, à la vie. David quant à lui, s'est approché de la mort pour se sentir exister. Ce rapport complexe de la dialectique vie-mort prend d'ailleurs sens dans une ordalie qui permet de jouer sa vie et sa mort.

\section{Bibliographie}

C. Adam, Jalons pour une théorie critique du Manuel diagnostic et statistique des troubles mentaux (DSM), 2012, Déviance et société, vol. 36, n² 2, pp. 137-169.

C. Adam, Psychopathologie et délinquance, 2015, Bruxelles, Bruylant.

American Psychiatric Association, Diagnostic and statistical manual of mental disorders : DSM-5, 2013, Washington, DC.
H. Becker, Outsiders : études de sociologie de la déviance, 1985, Paris, Métailié.

S. Brochu, Drogues et criminalité : point de vue critique sur les idées véhiculées, 1997, Déviance et société, vol. 21, n³, pp. 303-314.

M. Buber, Je et Tu, 2012, Paris, Éditions Aubier.

D. Burrick, Une épistémologie du récit de vie, 2010, Recherches qualitatives, $n^{\circ} 8$, pp. 7-36.

G. Canguilhem, Examen critique de quelques concepts : du normal, de l'anomalie et de la maladie, du normal et de l'expérimental, in G. Canguilhem, Le normal et le pathologique, 1975, Paris, PUF, pp. 76-95.

R. Castel, Les sorties de la toxicomanie, in A. Ogien, P. Mignon, La demande sociale de drogues, 1994, Paris, La documentation française, pp. 23-30.

N. das Neves Ribeiro, «Usager de drogues »: (dé)construction d'une figure de politiques pénales, 2018, Revue de science criminelle et de droit pénal comparé, vol. 2, n², pp. 569-580.

T. Decorte, Les effets adverses des politiques officielles en matière de drogue sur les mécanismes d'autorégulation des consommateurs de drogues illicites, 2010, Drogues, santé et société, vol. $9, \mathrm{n}^{\circ} 1, \mathrm{pp}$. 295-333.

P. Desfourneaux, Corps postmodernes et corps toxicomanes : quels rapports à l'enveloppe charnelle?, 2008, La clinique lacanienne, vol. 14, n² 2, pp. 195211.

M. Doyen, N. das Neves Ribeiro, Consommateur d'héroïne intégré socialement : un funambule social ?, 2019, Revue de droit pénal et de criminologie, $n^{\circ} 2$, pp. 140155 . 
A. Ehrenberg, M. Schiray, Penser la drogue, penser les drogues, 1992, Paris, Éditions Descartes.

C. Faugeron, M. Kokoreff, Société avec drogues : enjeux et limites, 2002, Ramonville Saint-Agne, Erès.

A. Fontaine, Double vie : les drogues et le travail, 2006, Paris, Les Empêcheurs de penser en rond.

A. Frances, Saving Normal. An Insider's Revolt Against Out-of-Control Psychiatric Diagnosis, DSM-5, Big Pharma, and the Medicalization of Ordinary Life, 2013, New York, William Morrow.

S. Freud, Notre relation à la mort, 2012, Paris, Payot \& Rivages.

V. de Gaulejac, Qui est « je »? Sociologie clinique du sujet, 2009, Paris, Seuil.

P. Hachet, La toxicomanie : du corps troué aux intrusions psychiques, 2005, Imaginaire \& Inconscient, vol. 16, $\mathrm{n}^{\circ} 2$, pp. 67-75.

M. Heidegger, Etre et temps, 1992, Paris, Gallimard.

D. Kaminski, Toxicomanie : le mot qui rend malade, 1990, Déviance et société, vol. 14, $\mathrm{n}^{\circ} 2$, pp. 179-196.

S. Kirk, H. Kutchins, Aimez-vous le DSM ? Le triomphe de la psychiatrie américaine, 1998, Le Plessis-Robinson, Institut Synthélabo pour le progrès de la Connaissance.

B. Lahire, L'homme pluriel : les ressorts de l'action, 1998, Paris, Nathan.

G. Lambrette, À son corps défendant. Une approche constructiviste du corps dans le champ des toxicomanies, 2008, Psychotropes, vol. 14, $n^{\circ} 2$, pp. 9-21.
D. Le Breton, Le contrecorps de la toxicomanie. Sémiotique d'addicts, 2012, Le sociographe, vol. $39, n^{\circ} 3$, pp. 55-64.

M. Marzano, La philosophie du corps, 2009, Paris, PUF.

L. Molitor, Les membres de services spécialisés de la police au prisme de l'image violente, e-legal, 2019, Revue de droit et de criminologie de l'ULB, vol. 3.

E. Mounier, Le personnalisme, 1961, Paris, PUF.

E. Mounier, Introduction aux existentialismes, 1962, Rennes, Presses Universitaires de Rennes.

C. Olievenstein, La vie du toxicomane, 1991, Paris, PUF.

J. Rhéaume, Quand l'histoire devient agissante. Étude de cas en milieu associatif, in V. de Gaulejac, M. Legrand, Intervenir par le récit de vie, 2008, Toulouse, Ed. Erès, pp. 6388.

P. Ricœur, Temps et récit. Tome 3 : Le temps raconté, 1985, Paris, Éditions du Seuil.

P. Ricœur, Soi-même comme un autre, 1990, Paris, Éditions du Seuil.

C. de Ryckel, F. Delvigne, La construction de l'identité par le récit, 2010, Psychothérapies, vol. 30, n 4, pp. 229240.

F. Toro, L'évacuation du plaisir : une question de rentabilité ? Première Partie, 1998, Les Cahiers de Prospective Jeunesse, vol. 3, n 4, pp. 1923.

C. Vauterin, Conduites suicidaires et criminologie, in M. Colin, Études de criminologie clinique, 1963, Paris, Ed. Masson, pp. 155-191. 